\title{
Digitize-PID: Automatic Digitization of Piping and Instrumentation Diagrams
}

\author{
Shubham Paliwal, Arushi Jain, Monika Sharma, Lovekesh Vig \\ TCS Research, Delhi, India \\ \{shubham.p3|arushi.jain|monika.sharma1|lovekesh.vig\}@tcs.com
}

\begin{abstract}
Digitization of scanned Piping and Instrumentation diagrams (P\&ID), widely used in manufacturing or mechanical industries such as oil and gas over several decades, has become a critical bottleneck in dynamic inventory management and creation of smart P\&IDs that are compatible with the latest CAD tools. Historically, P\&ID sheets have been manually generated at the design stage, before being scanned and stored as PDFs. Current digitization initiatives involve manual processing and are consequently very time consuming, labour intensive and error-prone. Thanks to advances in image processing, machine and deep learning techniques there is an emerging body of work on P\&ID digitization. However, existing solutions face several challenges owing to the variation in the scale, size and noise in the P\&IDs, the sheer complexity and crowdedness within the drawings, domain knowledge required to interpret the drawings and the very minute visual differences among symbols. This motivates our current solution called Digitize-PID which comprises of an end-to-end pipeline for detection of core components from P\&IDs like pipes, symbols and textual information, followed by their association with each other and eventually, the validation and correction of output data based on inherent domain knowledge. A novel and efficient kernel-based line detection and a two-step method for detection of complex symbols based on a fine-grained deep recognition technique is presented in the paper. In addition, we have created an annotated synthetic dataset, Dataset-PEID, of 500 P\&IDs by incorporating different types of noise and complex symbols which is made available for public use (currently there exists no public P\&ID dataset). We evaluate our proposed method on this synthetic dataset and a real-world anonymized private dataset of 12 P\&ID sheets. Results show that Digitize-PID outperforms the existing state-of-the-art for P\&ID digitization.
\end{abstract}

\section{Introduction}

A Piping and Instrumentation Diagram (P\&ID) is a standardized schematic illustration used in the process engineering industry to record mechanical equipment, piping, instrumentation and control devices employed in the physical implementation of a process. P\&IDs are created at the design stage of the process, stored in an image or PDF format and play an important role in the maintenance and modification stage of the physical process flow. Over the years, there are millions of PID sheets that have been manually generated, scanned and stored as images. The valuable information trapped in these images needs to be 
unlocked and integrated with modern smart P\&ID systems. This digitization is necessary to facilitate easy reuse of data and design, automate mundane tasks, maintain inventory, reduce time, increase efficiency and productivity. Currently, P\&ID sheets are manually processed by engineers which is a very burdensome, time consuming and error-prone task. There is a very high cognitive load involved in manual digitization due to the minor variations in symbols, scale, size and noise within the sheets, in addition to the crowdedness of text, symbols and line. There is also significant domain knowledge involved in determining line changes and associating text with lines and symbols. Extraction and analysis of textual information, pipelines, and symbols as graphic objects and shapes are the key tasks for interpreting P\&ID sheets. We exploit the recent advances in deep learning/machine learning for these tasks.

Several approaches have been proposed for digitizing P\&ID sheets or similar documents. This includes conversion of scanned engineering drawings into 3D representation CAD files [10], symbol recognition [3] and classification [1, and shape representation [17. Ishii et al. 8 presented work towards reading hand drawn piping and instrument diagram where lines, symbols and characters are separated hierarchically from the vectorized representation. In another paper by Gellaboina et al. [7], an iterative learning approach based on hopfield neural networks was presented to detect symbols in P\&ID sheets.

Over the last decade, researchers have applied dynamic programming, machine learning, deep learning and pattern recognition to automate the detection of lines, text, shapes from PDFs and/or scanned images. Nazemi et al. 13. presented a method for detecting and extracting mathematical expressions, alphanumeric symbols to generate MathML of the scanned documents. A thorough review of prior methods and a general framework for the digitization of complex engineering diagrams was proposed by Moreno-Garcia et al. [12. Fu et al. [6] described a visual recognition approach by leveraging CNNs for symbol recognition and methods like multi-scale sliding window and connected component analysis for automatic localization. A semi-automatic and heuristic based approach for symbol localization is proposed by Elyan et al. 4] which utilizes machine learning models like Random Forests, Support Vector Machines (SVM), and CNNs. Kang et al. 9] proposed a two-fold method comprising of extraction of relevant components from P\&IDs followed by a recognition step that compares the input sheet at various angles with the objects registered in the database. Very recently, Rahul et al. 14 proposed a novel end-to-end approach based on a combination of low-level vision techniques and deep learning networks like CTPN [15] and FCN [1] for digital interpretation of P\&ID sheets by yielding the process flow in a tree format. The shortcoming of approaches proposed in [14] is that it utilizes a hough transform for detecting lines which is parameter-dependent and does not perform well on noisy P\&IDs. Moreover, it uses CTPN for text detection which is not able to identify vertical text components present in P\&IDs.

Although significant efforts have been made to improve the performance of automatic methods for conversion of P\&IDs into digital drawing, but perfect automatic recognition is still not achievable [12. To this end, we propose an 


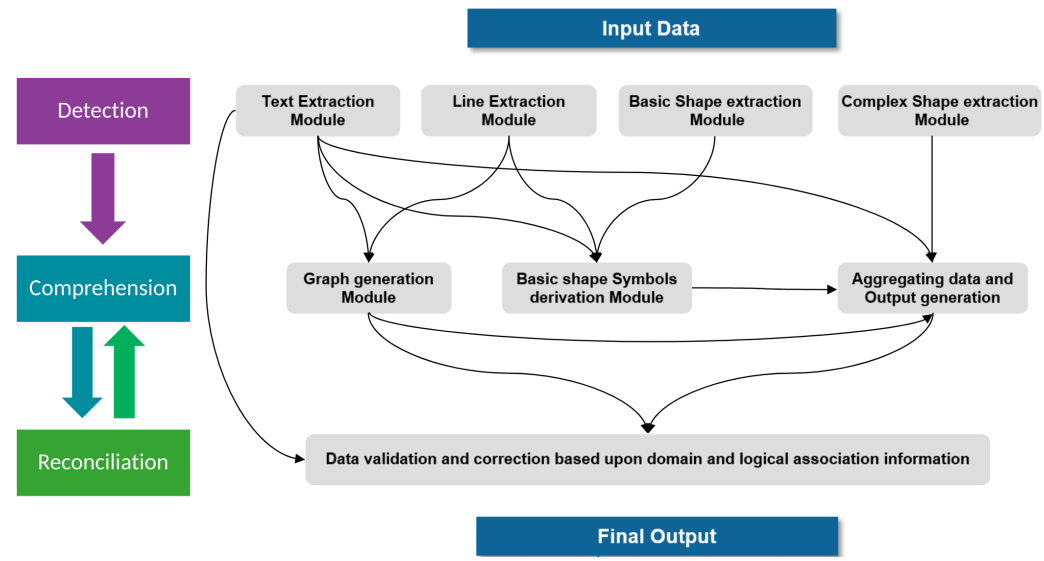

Fig. 1: An overview of Digitize-PID which consists of 3 sequential modules with their corresponding sub-modules: Detection, Comprehension and Reconciliation.

end-to-end pipeline called Digitize-PID which leverages computer-vision techniques and deep learning methods to first detect various components of interest such as lines, graphic symbols and textual information; followed by their aggregation and association with each other; and finally, validation and correction of the extracted output data based on domain rules. We describe a robust kernelbased approach for line detection which works well even in noisy environments. Additionally, a two-step process for detecting complex symbols having minute differences in visual structure is presented which utilizes a deep learning based network for symbol localization and fine-grained classification. We evaluate the effectiveness of our proposed solution on a real-world dataset of 12 P\&ID sheets and show impressive results. Note that while 12 may seem like a small number, each P\&ID sheet is a very high resolution image with hundreds of visual and textual components. Since, there exists no publicly available dataset for P\&ID sheets, we synthesize our own synthetic dataset named Dataset-PESID ${ }^{\text {T }}$. We also benchmark this dataset using Digitize-PID and make it publicly available for accelerating community advances in this field. To summarize, our key contributions in this paper are:

- We propose Digitize-PID, an end-to-end novel and robust pipeline for digitizing P\&ID sheets by leveraging computer vision and deep learning.

- Digitize-PID combines novel image-processing techniques for hard low-level vision problems such as line detection, dashed line detection, corner detection and a deep learning pipeline for symbol detection and recognition.

- We create a synthetic dataset of P\&ID sheets called Dataset-P\&ID consisting of $500 \mathrm{P} \& I D$ sheets with corresponding annotations for training and evaluation purposes. The dataset is released online for public use.

1 https://drive.google.com/drive/u/1/folders/1gMm_YKBZtXB3qUKUpI-LF1HE_ MgzwfeR 
- We benchmark our proposed solution Digitize-PID on two datasets: a realworld dataset of 12 P\&IDs and a synthetic Dataset of 100 P\&IDs, and present the results in Section 4

- We also compared the performance of Digitize-PID against prior state-ofthe-art methods by Rahul et al. 14] and outperformed it.

The remaining sections of the paper are structured as follows: Section 2 describes the problem statement and discusses about the detection, comprehension, and reconciliation steps of our proposed pipeline. Section 3 provides details about the synthetic dataset that we have generated for training and evaluation purposes. This is followed by experimental details and results in Section 4 . Finally, we conclude the paper in Section 5.

\section{Proposed Method: Digitize-PID}

In this paper, the task is to automate the process of P\&ID digitization to convert the scanned legacy P\&ID sheets into a structured format. The proposed method should be capable of identifying different industrial components such as symbols, pipes along with their labeled text and neighbouring symbols. The proposed pipeline Digitize-PID takes an input P\&ID image and outputs a .csv file consisting of two separate tables listing - (1) different instances of symbols with their mapped text labels and connected pipelines; and (2) containing the list of inter-connectivity between different pipelines representing a graph.

Digitize-PID consists of three high level steps: Detection, Comprehension and Reconciliation, as shown in Figure 1. The Detection step involves extraction of different components from P\&ID sheets such as text, lines and symbols which are essential to execute the subsequent steps in the pipeline. The Comprehension step consists of logically aggregating the different components detected in the previous stage, for example, the graph generation module takes basic features like lines, symbols and textual information as input and associates the appropriate symbols and text to lines. Finally, the Reconciliation step comprises of applying different domain/business rules and final corrections/tweaks on the output data of the comprehension stage. Next, we present a detailed description of these 3 steps in the following sub-sections.

\subsection{Detection}

The detection module comprises of the following sub-modules, as shown in Figure 2, which are independent and executed in parallel.

- Text Extraction module: P\&ID sheets contain text for labeling different components and specifying different parameters of pipelines. We perform text extraction via a 2 -step process which involves dividing a P\&ID image into multiple fixed-sized overlapping patches. These patches are processed using a Character Region Awareness for Text Detection (CRAFT) 2] network, which predicts bounding boxes (Bbox) for text regions. CRAFT works robustly even for vertically aligned texts. The overlapping Bboxes across overlapping patches are merged using IOU metric which helps to localize the text 

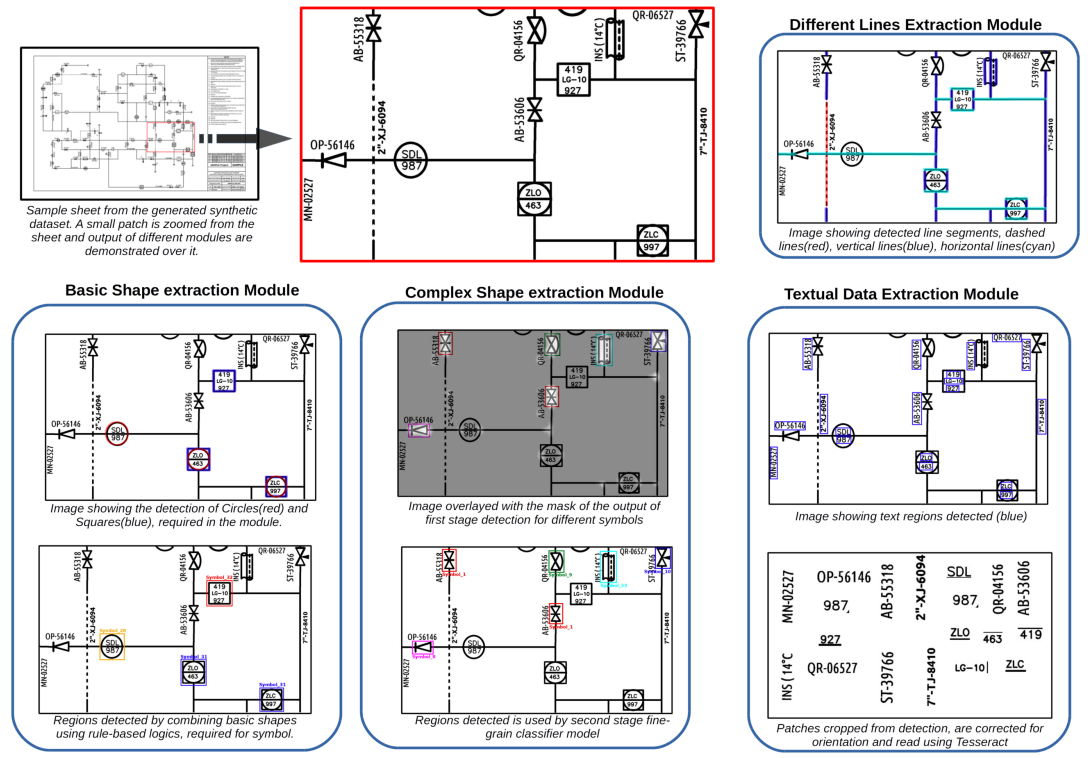

Fig. 2: Figure illustrating different sub-modules of the Detection step of DigitizePID over a sample P\&ID sheet (a small image-patch is zoomed for visual clarity).

with high accuracy and effectively reduces the cases of missing texts. These merged Bboxes are projected on the input P\&ID sheet and text-patches are extracted. These patches contain single-lined texts read using Tesseract.

- Line Extraction module: A P\&ID sheet utilizes a network of different types of lines to denote connections between different components, which collectively represent the desired process flow. In Digitize-PID method, we perform line detection using filters based on a structuring element matrix. In the pixel representation, a line can be defined as set of continuous adjacent points in a particular orientation (line orientation). Thus, even an infinitesimal segment of a line can be seen as a basic building block for the entire line. A structuring element is defined as a binary matrix of a fixed dimension $(m \times n)$, in which all active regions denote the filtering line's infinitesimal segment. However, for practical purposes, we do not choose an infinitesimal segment for a structuring element matrix, rather we choose a size greater than the line width and as a function of image spatial resolution, so as to avoid noise and scaling effects in line detection.

Formally, lets assume a binary image $\boldsymbol{A}$ as an integer grid $\boldsymbol{Z}^{d}$ of dimension $d$ (here $d=2$ ), and $\boldsymbol{B}$ is the line structuring element belonging to the same set $\boldsymbol{Z}^{d}$. We first perform erosion on $\boldsymbol{A}$ using $\boldsymbol{B}$, as given in Eqn. 1. As a result, we filter out all the elements not resembling $\boldsymbol{B}$. Next, the filtered regions in the image are restored by performing a dilation operation, as given in Eqn. 2 , using the same structuring element $\boldsymbol{B}$.

$$
\begin{gathered}
\boldsymbol{A}^{\text {erode }}=\min _{\left(x^{\prime}, y^{\prime}\right): B\left(x^{\prime}, y^{\prime}\right) \neq 0} \boldsymbol{A}\left(x+x^{\prime}, y+y^{\prime}\right) \\
\boldsymbol{A}^{\text {dilate }}=\max _{\left(x^{\prime}, y^{\prime}\right): B\left(x^{\prime}, y^{\prime}\right) \neq 0} \boldsymbol{A}^{\text {erode }}\left(x+x^{\prime}, y+y^{\prime}\right)
\end{gathered}
$$




$$
\operatorname{conv}(P)=\left\{\sum_{i=1}^{n} \lambda_{i} p_{i} \mid \sum^{n} \lambda_{i}=1 \wedge \forall i \in\{1, . ., n\}: \lambda_{i} \geq 0 \wedge p_{i} \in P\right\}
$$

Subsequently, the pixé $\bar{F}^{1}$ obtained in the activated regions generate different contours over line regions. Each contour formed over set $P$, containing $n$ pixel points, is bounded by a convex hull conv $(P)$. The convex hull conv $(P)$, as defined in Eqn. 3, is the intersection of all convex supersets of $P$ [16], which ensures a tight bound over the convex contour of the line. Finally, the two extreme end points from the set $\operatorname{conv}(P)$ are computed, along the orientation of the structuring element and are treated as end points of the detected line.

Dashed Line detection: Here, we are focusing on Dashed Lines present in P\&IDs which are a series of line segments separated by equal distance, as shown in Figure 2. We leverage the collinearity and consistency properties of dashed lines for detection. There are two thresholds that we use for segmentlength and distance between consecutive line-segments (gaps) which are determined based on the average value for the line cluster having the least mean segment-length and gap. The cases of jumps in the series are very often noticeable in P\&ID sheets which lead to inconsistency in gaps between segments of dashed lines. This consistency is retained by applying a rule for filtering out contiguous jumps (three or more). The detected series are then merged to form the continuous chain. The only candidates for merging are the series of segments with opposite orientation and in close proximity with each other which are obtained using the DBSCAN [5] algorithm.

- Basic Shape extraction module: Among various symbols used in P\&ID sheets, certain symbols are composed of primitive shapes, such as rectangles and circles (Figure 3). Some of these symbols are differentiated via the texts written inside them. One such basic shape is a circle which are detected by applying Hough transforms across different overlapping image patches followed by their aggregation.

- Complex Shape extraction module: P\&ID sheets also contain very complex symbols whose structures have very minute inter-class differences and are difficult to interpret and derive via traditional image-processing. These symbols are detected using a 2-step process which consists of a deep learning pipeline for symbol localization followed by fine-grained recognition. As evident in Figure 3 . most of the complex symbols have very similar shape, thus it is preferable to create a common class for all such symbols for symbol localization. For this, we have trained an FCN [11] based semantic segmentation model which is used to localize all such symbols. We apply this FCN model to obtain region-proposals for symbols which are subsequently fed as input to a TBMSL-Net [18] network trained for fine-grained symbol classification.

\subsection{Comprehension}

Now, we describe how we derive many essential properties of P\&IDs by using the appropriate logical combination of text, symbols and lines obtained previously. 


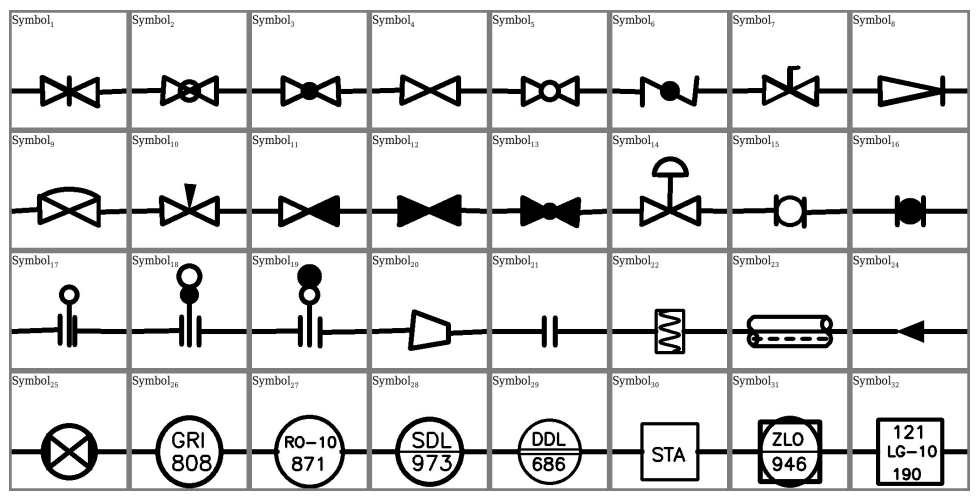

Fig. 3: Figure showing a set of 32 different symbols used for Dataset-P\&ID. Symbol $_{1}$ to Symbol $_{25}$ are complex symbols as they are structurally very similar and are detected using a Complex Shape Extraction module. Remaining Symbol $_{26}$ to Symbol $_{32}$ are detected using the Basic Shape Extraction module.

- Graph generation: The interactions between different components of P\&ID are represented by a web of lines that can be interpreted as a weighted graph structure. The graph representation assumes that the components are vertices and the connecting lines between components are edges. The connecting edges are of varying shapes, which can be decomposed into a combination of multiple straight lines of arbitrary lengths which enables us to create a graph with all straight line edges. For graph generation, we utilize the line information extracted in the detection step to filter out the lines with length smaller than the resolution-dependent threshold $(\alpha)$. Similarly, we remove the lines overlapping with regions of texts/symbols. The remaining lines (let's say $n$ ) are taken as edges, and the two end-points of each line are taken as vertices. This creates $n$ separate graphs, each having 2 vertices. Thus, the junction centers having $k$ lines will have $k$ vertices, occurring in close proximity, and another $k$ vertices pointing away in different directions. These neighbouring vertices at the junction points can be interpreted as separate clusters such that for each cluster, distance of its respective vertices from its mean (i.e., mean of vertices) would not exceed the line threshold value $\eta \alpha$ (where $0<\eta<1$ ). After optimizing cluster centers with respect to the vertices, we replace the cluster vertices with their respective cluster centers, thus aggregating the separate single-edged graphs to form a common graph.

After the graph structure is created, we assign labels to the edges. Generally, the edges labels are filtered out from the detected text using regular expressions provided by domain experts or manually by visual inspection. After the relevant labels are extracted, they are localized to corresponding graph edges which have the minimum euclidean distance. Finally, these labels on the edges are propagated using Breadth First Search, to the adjoining edges (computed for edges from left to right) with the additional stopping condition of not propagating over label-assigned edges. 
- Basic Shape Symbol detection: For extracting rectangular shapes, we use the vertex sampling method to obtain candidate vertices of possible rectangular regions, which are later verified as rectangles via their geometric properties. The vertex points are obtained by applying the morphological $A N D$ operator over the images of vertical and horizontal lines. Further verification of rectangle shapes is done by using the pixel values to satisfy the presence of edges across vertices. Finally, shapes which are different combinations of lines, circles and squares are logically assembled and localized. For cases where multiple symbols have the same shape, we use the embeddedtext to differentiate them. These texts circumscribed by the symbols also represent their labels.

- Data Aggregation: In this step, different components which include textual, graphical and symbolic information (including both complex and simple symbols) are aggregated such that each detected symbol is mapped to its label, graph nodes, and a separate identification ID is assigned. This helps to create a database of P\&ID symbols with their respective properties. Symbol mapping with graph vertices is done by using the nearest neighbour with Euclidean distance as metric. However, a similar approach cannot be used for text boxes as they are of arbitrary length and texts found using the mean will not necessarily be closest. Thus, $k$-nearest neighbors are computed to get $k$ nearest text boxes corresponding to each symbol. Among these $k$ words, either the regex provided by domain experts are used, or else consistency in the pattern of labels is optimized over all the symbols. The symbol labels pattern which are consistent over other symbol instances in P\&ID sheet are finally assigned to the symbol.

\subsection{Reconciliation}

The digitized data obtained from Comprehension step, are the final output of our proposed method. However, to address any errors/failure, we use the reconciliation step which validates and performs corrections according to domain/business rules. For example, in some arbitrary case, if the particular symbol's label has a static common name over the entire sheet, then the obtained associated text has to be re-validated and overwritten. Multiple iterations involving reconciliation steps can dramatically improve the accuracy of the proposed method even in the customized business scenarios.

\section{Dataset}

Since, there exists no publicly available dataset for P\&ID sheets, we have generated our own synthetic dataset named Dataset-PEID for training and evaluation purposes. Dataset-P\&ID consists of 500 annotated P\&ID sheets with a 4:1 traintest ratio and is made publicly available for the benefit of research community. It includes 32 different symbols, as given in Figure 3 , uniformly plotted over different graph structures which have been generated to mimic real world P\&ID sheets as we have introduced different types of noise such as pixelation, blurring, 
Table 1: Table showing performance of Symbol Recognition on Dataset-P\&ID. (Bottom-right) Figure showing the confusion matrix of complex symbols detected using proposed deep learning pipeline on Dataset-P\&ID.

(a) Complex Symbols

\begin{tabular}{cccc} 
Symbol & Precision & Recall & F1-score \\
\hline 1 & 0.932 & 0.882 & 0.906 \\
2 & 0.968 & 0.968 & 0.968 \\
3 & 0.965 & 0.847 & 0.902 \\
4 & 0.974 & 0.904 & 0.938 \\
5 & 0.986 & 0.973 & 0.979 \\
6 & 0.978 & 0.967 & 0.972 \\
7 & 0.971 & 0.911 & 0.940 \\
8 & 0.823 & 0.963 & 0.888 \\
9 & 0.772 & 0.986 & 0.866 \\
10 & 0.974 & 0.958 & 0.966 \\
11 & 0.741 & 0.991 & 0.848 \\
12 & 0.875 & 0.793 & 0.832 \\
13 & 0.972 & 0.938 & 0.955 \\
14 & 0.916 & 0.961 & 0.938 \\
15 & 0.947 & 0.997 & 0.971 \\
16 & 0.979 & 0.941 & 0.960 \\
17 & 0.813 & 0.979 & 0.888 \\
18 & 0.946 & 0.993 & 0.969 \\
19 & 0.946 & 0.724 & 0.820 \\
20 & 0.962 & 0.929 & 0.945 \\
21 & 0.876 & 0.988 & 0.929 \\
22 & 0.936 & 0.946 & 0.941 \\
23 & 0.881 & 0.956 & 0.917 \\
24 & 0.977 & 0.965 & 0.971 \\
25 & 0.927 & 0.743 & 0.825 \\
\hline & & &
\end{tabular}

(b) Basic Shape Symbols

\begin{tabular}{cccc}
\multicolumn{3}{c}{ Symbol Precision Recall } & F1-score \\
\hline 26 & 0.893 & 0.937 & 0.914 \\
27 & 0.864 & 0.903 & 0.883 \\
28 & 0.961 & 0.975 & 0.968 \\
29 & 0.977 & 0.984 & 0.980 \\
30 & 0.890 & 0.912 & 0.901 \\
31 & 0.904 & 0.892 & 0.898 \\
32 & 0.923 & 0.948 & 0.935 \\
\hline
\end{tabular}
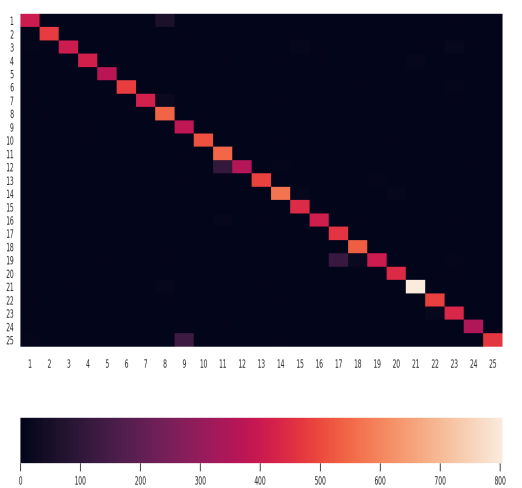

salt and pepper noise in the generated sheets. The labels are assigned to symbols and pipelines maintaining the standards followed for real world P\&ID. The ground-truth of the dataset consists of spatial information of symbols along with associated text labels and their connected pipeline. We provide sets of horizontal and vertical lines with their coordinates and a separate list containing all the texts present in P\&ID sheets along with their spatial position.

\section{Experimental Results and Discussions}

Here, we present the system configuration used for conducting experiments followed by the performance evaluation of Digitize-P\&ID. The performance is evaluated based on Recall, Precision and F1-score for different symbols taken over the test-split. A correct prediction of a symbol includes precise localization of symbol with $I O U>0.75$, symbol class and its associated text-label. Similarly, the output graph is evaluated based on the accuracy of correct adjacency list. However, the validation of graph-creation depends on domain information and is performed as part of the reconciliation step. 
(a) Setup: We have validated and refined our proposed pipeline via repeated experiments to identify optimal parameters. To begin with, we resize the images to have width of 7168 pixels while maintaining the aspect-ratio. In the detection module, for text detection we split the image into multiple square patches of dimension 800 pixels such that there is an overlap of $50 \%$ with their adjacent patches. The common text regions are segregated and read by using Tesseract with line configuration. The same process is also applied on the image by rotating it to capture missing vertical text. Next, we process the entire image at once for line detection. As mentioned earlier, the choice of kernel length is taken as $0.1 \%$ of the maximum image resolution. Similarly, in the Basic shape extraction module, the choice of range of radius for hough circle detection is also taken between $0.05 \%$ and $0.01 \%$ of maximum image resolution. Further, in case of complex shape detection, the image is processed at patch level with patches of size 400px. The output of the FCN model is filtered with threshold probability of 0.8 . Finally, the recognition threshold of TBMSL-Net is taken as 0.9 for identifying a symbol from a region-of-interest of image. In the comprehension step, the graph is generated as explained earlier, with the DBSCAN threshold of 50 and the neighbour threshold of 2 . The pipeline labels are spread across different pipelines using the Breadth first search algorithm. In data aggregation, we use the standard approach of connecting the nearest line entity. However for texts, we used 5 nearest neighbours, followed by the mapping in accordance with the symbol label rule provided in the reconciliation step. In the absence of such rules, the nearest label texts following the pattern is determined as the associated texts.

(b) Results \& Discussion: We first present the overall performance of symbol detection with correct associations on synthetic Dataset-P\&ID in Table 1a and Table $(1 \mathrm{~b})$ for the complex and basic shape symbols, respectively. We also show the confusion matrix to demonstrate the robustness of our proposed complex symbol detection module on the synthetic dataset. We use the nearest associated text to resolve the conflict of multiple symbols.

Table 2: Comparison of Digitize-P\&ID with prior-art [14] on 12 real-world P\&IDs

\begin{tabular}{|l|l|l|l|l|c|c|}
\hline & \multicolumn{2}{|c|}{ Precision } & \multicolumn{2}{c|}{ Recall } & \multicolumn{2}{c|}{ F1-score } \\
\hline Symbols & {$[\mathbf{1 4}]$} & Ours & {$[\mathbf{1 4}]$} & \multicolumn{1}{|c|}{ Ours } & {$[\mathbf{1 4}]$} & Ours \\
\hline Bl-V & 0.925 & $\mathbf{0 . 9 6 3}$ & 0.936 & $\mathbf{0 . 9 8 6}$ & 0.931 & $\mathbf{0 . 9 7 4}$ \\
Ck-V & 0.941 & $\mathbf{0 . 9 6 8}$ & 0.969 & $\mathbf{0 . 9 8 8}$ & 0.955 & $\mathbf{0 . 9 7 8}$ \\
Ch-sl & $\mathbf{1 . 0 0 0}$ & 0.990 & 0.893 & $\mathbf{0 . 9 4 6}$ & 0.944 & $\mathbf{0 . 9 6 7}$ \\
Cr-V & 1.000 & 1.000 & $\mathbf{0 . 9 8 9}$ & 0.973 & 0.995 & $\mathbf{0 . 9 8 6}$ \\
Con & $\mathbf{1 . 0 0 0}$ & 0.975 & 0.905 & $\mathbf{0 . 9 4 0}$ & 0.950 & $\mathbf{0 . 9 5 7}$ \\
F-Con & 0.976 & 0.976 & 0.837 & $\mathbf{0 . 9 0 5}$ & 0.901 & $\mathbf{0 . 9 3 9}$ \\
Gt-V-nc & 0.766 & $\mathbf{0 . 8 6 4}$ & 1.000 & 1.000 & 0.867 & $\mathbf{0 . 9 2 7}$ \\
Gb-V & 0.888 & $\mathbf{0 . 9 1 3}$ & 0.941 & $\mathbf{0 . 9 4 6}$ & 0.914 & $\mathbf{0 . 9 2 9}$ \\
Ins & 1.000 & 1.000 & $\mathbf{0 . 9 8 5}$ & 0.964 & $\mathbf{0 . 9 9 2}$ & 0.982 \\
GB-V-nc & 1.000 & 1.000 & 0.929 & $\mathbf{0 . 9 3 6}$ & 0.963 & $\mathbf{0 . 9 6 7}$ \\
Others & 0.955 & $\mathbf{0 . 9 7 3}$ & $\mathbf{1 . 0 0 0}$ & 0.990 & 0.970 & $\mathbf{0 . 9 8 1}$ \\
\hline
\end{tabular}


Next, we compare our results with Rahul et.al [14. The symbol detection accuracy is compared on the same set of symbols, used in [14, on the 12 real P\&ID sheets dataset as given in Table 2. It shows that the F1-score of complex symbol detection module of Digitize-PID is better than prior art 14]. Please note that the experiment is conducted with all the symbols, but the network is only trained to identify the concerned symbols and the remaining symbols are grouped into an 'others' class. We also illustrate that our proposed structuring element based line detection is more robust than the hough line detection used in 14, as shown in Figure 4. Finally, we present the performance of text detection and recognition on our dataset in Table $3 \mathrm{a}$. The IOU threshold value is used to find texts having significant overlap with ground truth which are used for further recognition. Since the text-labels contain very critical information, we consider a prediction to be correct only when there is an exact match with the ground-truth label. Table 3b shows the accuracy for line detection, for both complete (99.34\%) and dashed (82.91\%) lines (since line detection is not-learning based, results are computed over entire dataset).

Table 3: Performance of Digitize-PID on synthetic Dataset-P\&ID

(a) Performance of Text Detection and Recognition module

\begin{tabular}{lll} 
IOU & $A c c_{\text {Det }}$ & $A c c_{\text {Rec }}$ \\
\hline$<0.9$ & $87.18 \%$ & $79.21 \%$ \\
\hline
\end{tabular}

(b) Performance of Dashed and Complete Line Detection module

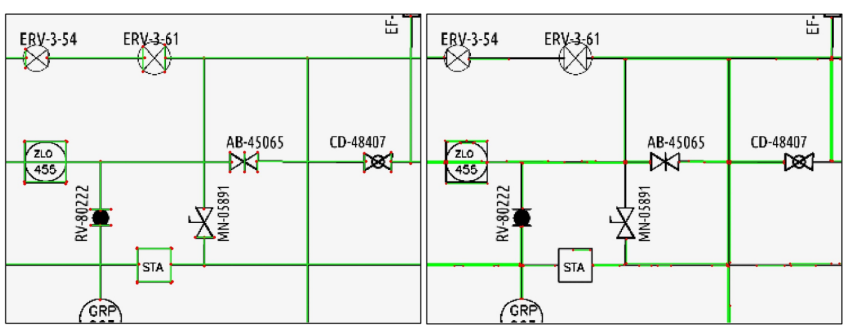

Fig. 4: Left image shows our structuring element based line-detection output and Right image shows hough line-detection [14] output.

\section{Conclusion}

In this paper, we have proposed a complete automated pipeline for extracting relevant information from P\&IDs, which are commonly used across several industry verticals for depicting a formal process flow. The proposed pipeline, named Digitize-PID utilizes a combination of state-of-the-art methods for text recognition, robust line detection using morphological operations and a two-step deep-learning based pipeline for fine-grained symbol detection and recognition. 
Finally, we combine all the extracted information in a graph and organize the extracted data into database-compatible tables. In addition to this, we have synthesized a dataset for P\&IDs (Dataset-P\&ID) along with their ground-truth annotations which is made public for validation by other researchers.

\section{References}

1. Ablameyko, S., Uchida, S.: Recognition of engineering drawing entities: Review of approaches. International Journal of Image and Graphics 7, 709-733 (10 2007)

2. Baek, Y., Lee, B., Han, D., Yun, S., Lee, H.: Character region awareness for text detection (craft). Conference on Computer Vision and Pattern Recognition (CVPR) (2019), https://arxiv.org/abs/1904.01941

3. Cordella, L., Vento, M.: Symbol recognition in documents: A collection of techniques? IJDAR 3, 73-88 (12 2000). https://doi.org/10.1007/s100320000036

4. Elyan, E., Garcia, C.M., Jayne, C.: Symbols classification in engineering drawings. In: 2018 International Joint Conference on Neural Networks (IJCNN). pp. 1-8 (2018). https://doi.org/10.1109/IJCNN.2018.8489087

5. Ester, M., Kriegel, H.P., Sander, J., Xu, X.: A density-based algorithm for discovering clusters in large spatial databases with noise. In: Proceedings of the Second International Conference on Knowledge Discovery and Data Mining. p. 226-231. KDD'96, AAAI Press (1996)

6. Fu, L., Kara, L.: From engineering diagrams to engineering models: Visual recognition and applications. Computer-Aided Design 43, 278-292 (03 2011). https://doi.org/10.1016/j.cad.2010.12.011

7. Gellaboina, M., Venkoparao, V.: Graphic symbol recognition using auto associative neural network model. pp. 297-301 (02 2009). https://doi.org/10.1109/ICAPR.2009.45

8. Ishii, M., Ito, Y., Yamamoto, M., Harada, H., Members, M.: An automatic recognition system for piping and instrument diagrams. Systems and Computers in Japan 20, 32 - 46 (09 2007). https://doi.org/10.1002/scj.4690200304

9. Kang, S.O., Lee, E.B., Baek, H.K.: A digitization and conversion tool for imaged drawings to intelligent piping and instrumentation diagrams (p\&id). Energies 12, 2593 (07 2019). https://doi.org/10.3390/en12132593

10. Kanungo, T., Haralick, R.M., Dori, D.: Understanding engineering drawings: A survey

11. Long, J., Shelhamer, E., Darrell, T.: Fully convolutional networks for semantic segmentation. CoRR abs/1411.4038 (2014), http://arxiv.org/abs/1411.4038

12. Moreno-garcía, C., Elyan, E., Jayne, C.: New trends on digitisation of complex engineering drawings. Neural Computing and Applications 31(6), 1695-1712 (Jun 2019). https://doi.org/10.1007/s00521-018-3583-1

13. Nazemi, A., Murray, I., Mcmeekin, D.: Mathematical information retrieval (mir) from scanned pdf documents and mathml conversion. IPSJ Transactions on Computer Vision and Applications 6, 132-142 (01 2014). https://doi.org/10.2197/ipsjtcva.6.132

14. Rahul, R., Paliwal, S., Sharma, M., Vig, L.: Automatic information extraction from piping and instrumentation diagrams. In: ICPRAM (2019)

15. Tian, Z., Huang, W., He, T., He, P., Qiao, Y.: Detecting text in natural image with connectionist text proposal network (2016)

16. Weisstein, E.W.: "convex hull.". From MathWorld-A Wolfram Web Resource. https://mathworld.wolfram.com/ConvexHull.html 
17. Zhang, D., Lu, G.: Lu, g.: Review of shape representation and description techniques. pattern recognition 37, 1-19. Pattern Recognition 37, 1-19 (01 2004). https://doi.org/10.1016/j.patcog.2003.07.008

18. Zhang, F., Zhai, G., Li, M., Liu, Y.: Three-branch and mutil-scale learning for finegrained image recognition (tbmsl-net). arXiv preprint arXiv:2003.09150 (2020), http://arxiv.org/abs/2003.09150 